\title{
From the FDA
}

\section{Botulism warning on garlic-in- oil mixes}

Because of a botulism risk, the FDA has ordered companies to stop making garlic-in-oil mixes that require refrigeration for safety. The agency also urges consumers to discard these and similar homemade products.

To be safe, the garlic-in-oil products should contain additional ingredients-specific levels of microbial inhibitors or acidifying agents such as phosphoric or citric acid. Most garlic products do contain these additives, as disclosed on their labels. However, some specialty mixes do not include the additives and need to be refrigerated from production through use. The FDA is notifying manufacturers, repackers, food distributors, and retailers to withdraw these products.

FDA Commissioner Frank E. Young, MD, PhD, says, "FDA's concern is that many consumers and retailers may consider such products to be nonperishable so they don't maintain them under adequate refrigeration." Homemade mixes can develop botulism, too. Dr Young warns that these home products should be made fresh for each use and not left out at room temperatures.

When two men and a woman from Kingston, NY, were hospitalized with botulism after eating a commercial mix that had not been refrigerated, the FDA at first issued a warning to consumers that the improperly formulated products left at room tem- perature may develop the deadly botulism toxin. But the agency reviewed these products' safety further and decided to eliminate the susceptible mixes.

The FDA says the studies showed that unrefrigerated garlic-in-oil mixes lacking antimicrobial agents can permit the growth of Clostridium botulinum bacteria with subsequent toxin production without affecting the taste and smell of the products. It also showed that the toxin production can occur even when a small number of $C$ botulinum spores are present in the garlic. When the spore-containing garlic is covered with oil and bottled, an oxygen-free environment is created that promotes the germination of spores and the growth of microorganisms at temperatures as low as $50^{\circ} \mathrm{F}$.

\section{Safety alert on sodium azide contamination in hemodialysis}

The FDA has issued a safety alert to hemodialysis centers and contractors who provide water for these centers. There is a potentially hazardous contamination problem that can occur if dialysis water treatment filters preserved with sodium azide are not adequately rinsed before being used.

The alert by the FDA follows an incident in which nine patients experienced life-threatening hypotension attributed to contamination of the water treatment system with sodium azide from newly installed ultrafilters that had not been prerinsed before use. The filters had been packed in a preservative solution containing $0.25 \%$ sodium azide to prevent bacterial contamination and $25 \%$ glycerin to prevent desiccation.

The patients also had blurred vision, severe abdominal pain, headache, and loss of consciousness.

The safety alert contains instruction on adequate prerinsing for safe use of filters preserved with sodium azide. The manufacturer's directions should be followed closely and the manufacturer called if there are questions.

\section{Additional indication ap- proved for gemfibrozil}

The FDA has approved the lipidregulating agent, gemfibrozil (Lopid, Parke-Davis, Morris Plains, NJ), for use in reducing the risk of coronary heart disease in certain patients. The agent already had approval for treating patients with types IV or V hyperlipoproteinemia with high triglyceride levels who are at risk of pancreatitis, and who do not respond adequately to appropriate dietary therapy. The labeling notes that such patients commonly have fasting serum triglyceride levels greater than 2000 $\mathrm{mg} / \mathrm{dL}(22.60 \mathrm{mmol} / \mathrm{L})$ and that those with values under $1000 \mathrm{mg} /$ $\mathrm{dL}(11.3 \mathrm{mmol} / \mathrm{L})$ are unlikely to be at risk of pancreatitis.

The new indication notes that gemfibrozil can be considered in 
type IIB patients with low-density lipoprotein cholesterol (HDL) levels in addition to elevated low-density lipoprotein (LDL) cholesterol and elevated triglyceride levels and who have had an inadequate response to a weight reduction program, dietary therapy, exercise, and other pharmacologic agents, such as bile acid sequestrants and nicotinic acid, which are known to reduce LDL and raise HDL cholesterol levels.

The labeling further states that the potential benefit of gemfibrozil in treating type IIA patients with elevations of LDL cholesterol levels only is not likely to outweigh the risks: potential development of malignancy, gallbladder disease, and abdominal pain leading to appendectomy and other abdominal surgery.

To prevent bleeding complications, the labeling advises caution when anticoagulants are given in conjunction with gemfibrozil. It also describes a drug interaction resulting in rhabdomyolysis that has occurred when gemfibrozil and lovastatin have been administered concurrently.

The new labeling should be consulted for further details.

\section{From the NIH}

\section{Study shows effects of diet on breast cancer risk}

Reducing foods from animal sources may substantially decrease breast cancer risk, according to findings from a recent study in Italy (JNCI, $1989 ; 81: 278-286$ ). The researchew0 was supported in part by the National Cancer Institute and the National Institute of Environmental Health Sciences. The study was conducted in Italy, but the data were analyzed by Paolo Toniolo, MD, of the Department of Environmental Medicine, New York University Medical Center, New York, and associates.

They analyzed data on the intake of total fat, saturated fat, animal proteins, and other macronutrients in 250 women with breast cancer. The same data were studied in a control group of 499 women who represented a stratified random sample from the general population. Multivarite analysis showed that the relative risk of breast cancer was 3.0 for women in the highest quintile of consumption of saturated fat and 2.9 for those in the highest quintile of consumption of animal protein.

The researchers note that reducing fat intake to less than $30 \%$ of total calories, reducing saturated fat to less than $10 \%$ of the total calories, and reducing animal protein to less than $6 \%$ of total calories may have a strongly protective effect against breast cancer.

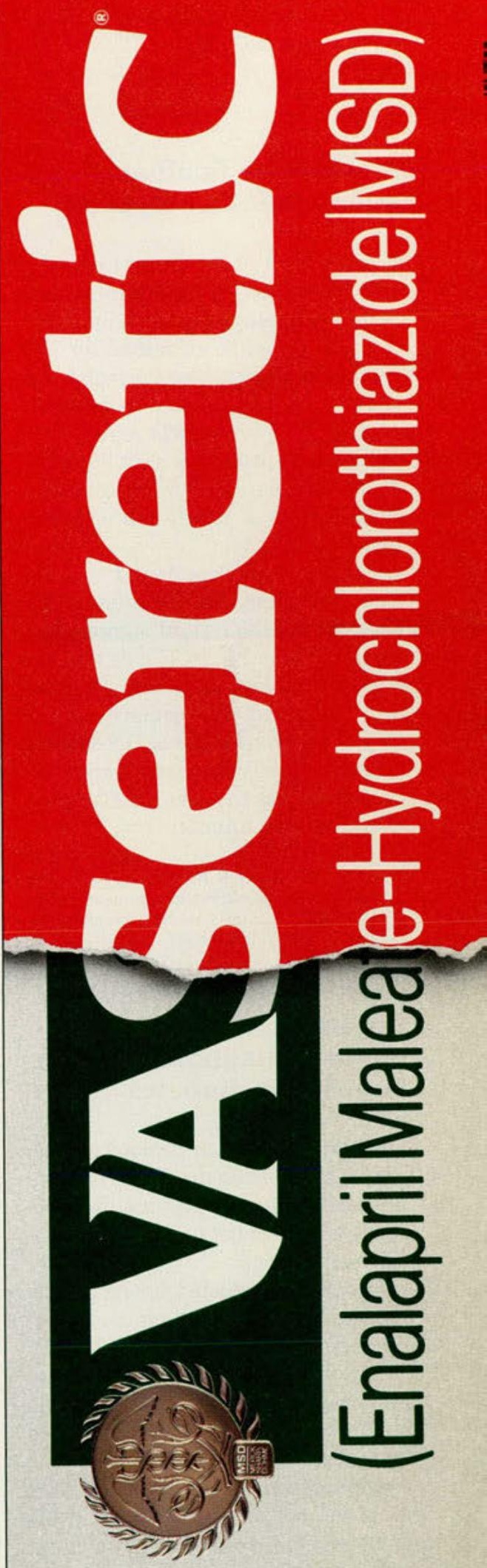

